\title{
Lack of Relationship of Egg White Intake with Occurrence of Leukopenia in Gynecologic Cancer Patients during Chemotherapy
}

\author{
Prapaporn Suprasert*, Apiwat Aue-Aungkul, Nuthaya Pautad
}

\begin{abstract}
Egg white intake during chemotherapy is common advice for cancer patients for the prevention of leukopenia. However, the benefit is uncertain. We conducted this prospective study to identify the relationship of egg white intake for gynecologic cancer patients who received carboplatin and paclitaxel and the occurrence of leukopenia. Between January 2014 and January, 2015, 81 patients were interviewed regarding their intake of egg whites before receiving subsequent chemotherapy. The basic data, the details of egg white intake and the grade of leukopenia in the previous cycle were recorded. The mean age was 54.1 years and $80 \%$ of the patients had a diagnosis of ovarian or endometrial cancer. The patients were interviewed at cycles 1-3 in 45 cases, 4-6 in 45 cases and 7-9 in two cases. Subsequent dose reduction was found in $6.2 \%$ and granulocyte-stimulating growth factors was given at $4.9 \%$. All the patients ate egg whites with variations in the number of eggs per day as follows: less than one (3), one to two (56), three to four (14) and five to six (8). Over $70 \%$ were recommended by nurses to eat egg whites and about $63 \%$ of patients received other supplemental food. Some $44.1 \%$ of the patients who ate less than or equal to two eggs per day and $36.4 \%$ who ate more than two eggs per day developed grade $2-4$ leukopenia, $\mathbf{P}=\mathbf{0 . 6 1}$. In conclusion, the data did not provide evidence in support of the conclusion that a greater egg white intake could significantly reduce the occurrence of leukopenia.
\end{abstract}

Keywords: Gynecological cancer - intake of egg whites - incidence of leukopenia - chemotherapy

Asian Pac J Cancer Prev, 17 (3), 1265-1267

\section{Introduction}

A common toxicity of gynecologic cancer patients who received chemotherapy was leukopenia especially in patients treated with paclitaxel plus carboplatin. This regimen is frequent used in various types of gynecologic cancers such as ovarian cancer, cervical cancer and endometrial cancer (Kim et al., 2012; Amadio et al., 2013; Markman, 2014). A previous publication reported leukopenia in over $40 \%$ of patients who received this regimen (Tas et al., 2013). Thus, the patients were given neutopenia precautions that included increasing the level of white blood cells by fully relaxing and eating only healthy foods. One of the most frequent food recommendations from healthcare personnel in our culture was to increase the level of white blood cells by white egg consumption. White eggs consist of proteins such as ovalbumin, ovotransferrin and ovamucoid that could provide anti-bacterial benefits and decrease the blood pressure (Constant, 2004; Miguel and Aleixandre, 2006). However, the property of increased white blood cells was demonstrated to increase only in piglets (Araki et al.,1993). Because there was no data of this benefit of regarding white egg intake and the occurrence of leukopenia in humans, we conducted this prospective study in gynecologic cancer patients who received a paclitaxel and carboplatin regimen.

\section{Materials and Methods}

After the proposal was approved by the local ethics committee, the gynecologic cancer patients scheduled to receive the next cycle of paclitaxel plus carboplatin (PT) regimen in the setting of first line treatment were invited to participate in this study. The patients with bowel obstruction or severe toxicity such as sepsis, severe emesis or who did not understand Thai language were excluded.

All participants who met the criteria were interviewed by our research team on the day they received chemotherapy before premedication administration. The details of egg consumption after receiving the previous cycle of chemotherapy such as the number of egg intake per day, 
the type of egg, and the cooking methods were recorded. The PT regimen consisted of paclitaxel at a dose of 175 $\mathrm{mg} / \mathrm{m} 2$ and carboplatin at the area under the curve of 5 given intravenously every three weeks. The patients were scheduled for evaluation of their bone marrow function by a complete blood count (CBC) at day 8, 12, 16 or according to the previous nadir day.

The granulocyte-stimulating growth factors (GCSF) were given when febrile neutropenia or grade 3,4 neutropenia occurred. All basic clinical data, WHO neutropenia grading, and the usage of GCSF were recorded and evaluated as descriptive parameters. The chisquare and Fisher Exact test were employed to compare the data. All of the data were analyzed using the SPSS version 17.0.

\section{Results}

Between January, 2014 and January, 2015, 81 patients were recruited to this study. The basic clinical data was noted in Table 1 . The mean age of patients was 54.12 years and about $50 \%$ were diagnosed with ovarian cancer. About $55 \%$ of the patients were interviewed during

Table 1. Basic Clinical Data $\mathbf{N}=81$

\begin{tabular}{|c|c|}
\hline & $\mathrm{N}(\%)$ \\
\hline Mean Age (Range: Year) & $54.12(32-79)$ \\
\hline \multicolumn{2}{|l|}{ Diagnosis } \\
\hline Cervix & $8(9.9)$ \\
\hline Corpus & $22(27.2)$ \\
\hline Ovary & $41(50.6)$ \\
\hline Tube & $4(4.9)$ \\
\hline PPA & $6(7.4)$ \\
\hline \multicolumn{2}{|l|}{ Cycle at Interview } \\
\hline $1-3$ cycle & $45(55.6)$ \\
\hline $4-6$ cycle & $45(55.6)$ \\
\hline 7 - 9 cycle & $2(2.4)$ \\
\hline Mean Body Mass Index $\left(\mathrm{kg} / \mathrm{m}^{2}\right)$ & $22.9(16.5-32.4)$ \\
\hline Mean Body Surface Area (m2) & $1.5(1.2-1.9)$ \\
\hline Underlying Disease & $18(22.2)$ \\
\hline DLP & 1 \\
\hline DM & 2 \\
\hline DM HT & 1 \\
\hline DM HT DLP & 1 \\
\hline HT & 9 \\
\hline HT DLP & 3 \\
\hline Heart & 1 \\
\hline Subsequent Dose Reduction & $5(6.2)$ \\
\hline GCSF Usage & $4(4.9)$ \\
\hline
\end{tabular}

DLP = dyslipidemia, DM = diabetic mellitus, $\mathrm{HT}=$ hypertension,

GCSF $=$ granulocyte colony stimulating factor received cycle $1-3$ of chemotherapy. The mean body mass index and body surface area was $22.9 \mathrm{~kg} / \mathrm{m} 2$ and $1.5 \mathrm{~m} 2$, respectively. About $6 \%$ of the studied patients required the dosage reduction of paclitaxel due to bone marrow toxicity and 5\% of the studied patients were given GCSF.

Regarding egg consumption behavior, the details were presented in Table 2. The majority of egg intake was reddish yellow and the most frequent cooking method was boiled egg. About one-third of the studied patients ate only white eggs and about one-fifth ate more than two eggs per day. The maximum egg intake per day was eight. Over $70 \%$ of the patients were advised to ingest eggs by the nursing team. Moreover, nearly $60 \%$ of the patients also ingested other supplemental food such as milk and vitamins.

Concerning the leukopenia in these patients, Grade 3 and 4 leukopenia toxicity was $28.4 \%$. The relationship of the number of egg intake and the occurrence of leukopenia was noted in Table 3. Grade 4 leukopenia occurred in patients who ate less than 1,1 to 2 and 5 to 6 eggs per day. The number of egg intake and the occurrence of leukopenia in each grade were not significantly related $(\mathrm{P}=0.35)$.

Table 2. Behavior of Egg White Intake

\begin{tabular}{lc}
\hline & $\mathrm{N}(\%)$ \\
\hline Type of Egg & \\
Duck Egg & $3(3.7)$ \\
Hen Egg & $78(96.3)$ \\
Cooking method & \\
Boiled & $70(86.4)$ \\
Mixed & $11(13.6)$ \\
Eaten Egg Whites Only & $26(32.1)$ \\
Number of Eggs Consumed per Day & \\
Less than 1 egg & $3(3.7)$ \\
1-2 eggs & $56(69.1)$ \\
3-4 eggs & $14(17.3)$ \\
5-6 eggs & $8(9.9)$ \\
Recommended By & \\
Nurse & $60(74.0)$ \\
Friend & $10(12.3)$ \\
Cousin & $8(9.9)$ \\
Book & $3(3.7)$ \\
Usage of other supplementary food & $51(63.0)$ \\
\hline
\end{tabular}

Table 3. Grade of Leukopenia

\begin{tabular}{lc}
\hline Grade & $\mathrm{N}(\%)$ \\
\hline 0 & $47(58.1)$ \\
2 & $11(13.6)$ \\
3 & $15(18.5)$ \\
4 & $8(9.9)$ \\
\hline
\end{tabular}

Table 4. Egg Intake and Grade of Leukopenia

\begin{tabular}{|c|c|c|c|c|c|c|}
\hline \multirow{2}{*}{$\begin{array}{l}\text { Number of Egg } \\
\text { Intake per Day }\end{array}$} & \multicolumn{5}{|c|}{ Grade } & \multirow{2}{*}{ Total } \\
\hline & 0 & 1 & 2 & 3 & 4 & \\
\hline Less than $1 \mathrm{egg}$ & $1(33.1)$ & - & - & - & $2(66.7)$ & 3 \\
\hline $1-2$ eggs & $32(57.1)$ & - & $9(16.1)$ & $10(17.9)$ & $5(8.9)$ & 56 \\
\hline $3-4$ eggs & $9(62.5)$ & - & $1(7.1)$ & $4(28.6)$ & - & 14 \\
\hline $5-6$ eggs & $5(62.5)$ & - & $1(12.5)$ & $1(12.5)$ & $1(12.5)$ & 8 \\
\hline Total & $47(58.0 \%)$ & - & $11(13.6 \%)$ & $15(18.5 \%)$ & $8(9.9 \%)$ & $81(100)$ \\
\hline
\end{tabular}




\section{Discussion}

The behavior of egg consumption in the present study really surprised our research team regarding the low intake of white eggs. Although all patients ate eggs, only $27 \%$ ate more than two eggs per day and only one-third of the studied patients ate only white eggs. To the best of our knowledge, no previous study has been published of the behavior of egg intake in other cancer patients. The present study was an initial study that showed the relationship of egg intake and the occurrence of leukopenia. Avian eggs have been found to be an important resource of many nutrients such as proteins, lipids, vitamins, minerals and growth factors as well as a number of defense factors to prevent bacterial and viral infection with a low price (Kovacs-Nolan et al., 2005). In the present study, the most frequent eggs preparation was boiled hen eggs. Regarding the prevention of leukopenia, Araki et al. (1993) reported the effect of oral administration of an active egg white product on neutrophil function in weanling piglets and found that piglets that received a dose of $30 \mathrm{mg} / \mathrm{kg} /$ day for a week showed increased neutrophil phagocytic activity. However, the data in humans is still lacking but white egg intake was frequently advised by health-care personnel to promote leukocytosis. In the present study, over $70 \%$ of the participants were recommended to intake white eggs for this purpose.

The prevalence of leukopenia in this study was about $40 \%$ that agreed with a previous report (Tas et al., 2013). In addition, the greater number of eggs consumed did not significantly decrease the occurrence of leukopenia. About one third of the patients who ate 5-6 eggs per day still developed leukopenia. The risk factors of leukopenia that increased the development of febrile neutropenia were elderly, poor performances status, advanced disease, low baseline blood cell counts, low body surface area and the type of chemotherapy regimen (Lyman et al., 2014).

Limitations of the present study were a limited number of studied patients and a study design that did not control other variable factors that might affect the prevalence of leukopenia as mentioned above except the type of chemotherapy regimen. However, this study was an initial study regarding the behavior of white egg consumption of Thai gynecologic cancer during chemotherapy and the effect on leukopenia.

In conclusion, white eggs consumption did not seem to be beneficial for preventing leukopenia. However, with the many inexpensive nutritional advantages (Kovacs-Nolan et al., 2005 ), white egg intake should be encouraged for patients during chemotherapy treatment.

\section{Acknowledgements}

We wish to thank the National Research University Project under Thailand's Office of Higher Education Commission and Chiang Mai University for the financial support in this project the pharmacotherapy for endometrial cancer. Expert Opin Pharmacother, 14, 2501-9.

Araki S, Kimura M, Suzuki M, et al (1993). Effect of active egg white product on neutrophil function in weanling piglets. $J$ Vet Med Sci, 55, 899-900.

Constant J (2004). The role of eggs, margarines and fish oils in the nutritional management of coronary artery disease and strokes. Keio J Med, 53, 131-6.

Kim A, Ueda Y, Naka T, et al (2012). Therapeutic strategies in epithelial ovarian cancer. J Exp Clin Cancer Res, 31,14-6.

Kovacs-Nolan J, Phillips M, Mine Y (2005). Advances in the value of eggs and egg components for human health.J Agric Food Chem, 53, 8421-31.

Lyman GH, Abella E, Pettengell R (2014). Risk factors for febrile neutropenia among patients with cancer receiving chemotherapy: A systematic review. Crit Rev Oncol Hematol, 90, 190-9.

Markman M (2014). Advances in cervical cancer pharmacotherapies. Expert Rev Clin Pharmacol, 7, 219-23.

Miguel M, Aleixandre A (2006). Antihypertensive peptides derived from egg proteins. $J$ Nutr, 136, 1457-60.

Tas F, Yildiz I, Kilic L, et al (2013). Same chemotherapy regimen leads to different myelotoxicity in different malignancies: a comparison of chemotherapy-associated myelotoxicity in patients with advanced ovarian and non-small-cell lung cancer. Am J Ther, 18, 1-3.

\section{References}

